International Tinnitus Journal. 2019;23(1):6-9.

\title{
Ossicular chain defects in adults with chronic otitis media
}

Arash bayat ${ }^{1,2}$

Nader Saki

Soheila Nikakhlagh ${ }^{1}$

Mohammad Amin Farshad

Mojtaba Lotfinia ${ }^{1}$

\begin{abstract}
Background and objective: Chronic otitis media (COM) is a common condition characterized by the perforation of the tympanic membrane and inflammation of the mucosal lining the hollow space in the middle ear and airy spaces of the temporal bone for at least 2-6 week. This study was carried out to find out the status of the middle ear ossicles in patients with COM and to correlate their status with clinical parameters.

Methods: This retrospective clinical study was conducted on $107 \mathrm{COM}$ patients (52 males and 55 females; age range: 18 to 75 years) submitted to surgery in the Otology Clinic at Imam Khomeini Hospital, Ahvaz Jundishapur University of Medical Sciences, Iran. Initially, a comprehensive case-history was obtained from patients and their hearing thresholds were recorded. Then, ossicles status and their junction condition were evaluated intraoperatively.
\end{abstract}

Results: The malleus was found intact in 70 (65.42\%), absent in 10 (9.34), and eroded in 27 (25.24\%) patients. Our results revealed that the incus was intact in $33(30.84 \%)$, eroded in $55(51.41 \%)$ and absent in $19(17.75 \%)$ subjects. Stapes was found intact in $54(50.46 \%)$ cases and eroded in 53 (49.54\%) cases. The mean Pure Tone Average (PTA) and Air-Bone Gap (ABG) comparisons in "intact" and "discontinuous" ossicular chain groups was not significant (Independent sample t-test, $\mathrm{p}>0.05$ ).

Conclusion: Our results demonstrated that incus was the most susceptible middle ear ossicle to erosion in COM, whereas the malleus was the most resistant ossicle. Furthermore, ABG and PTA values cannot be considered as a potential preoperative predictor for ossicular chain status.

Keywords: Ossicles, chronic otitis media, conductive hearing loss.

${ }^{1}$ Hearing Research Center, Imam Khomeini Hospital, Ahvaz Jundishapur University of Medical Sciences, Iran

${ }^{2}$ Department of Musculo skletal Rehabilitation Research Center, Ahvaz Jundishapur University of Medical Sciences, Ahvaz, Iran

*Send correspondence to:

Arash bayat

Department of hearing research center, Imam Khomeini Hospital, Ahvaz Jundishapur university of medical sciences Iran, Email: bayat-a@ajums.ac.ir Phone: +98

9183615157

Paper submitted to the ITJ-EM (Editorial Manager System) on November 20, 2018; and accepted on March 14, 2019. 


\section{INTRODUCTION}

Chronic Otitis Media (COM) is a pathological condition characterized by tympanic membrane perforation, varying degree of hearing loss and otorrhea lasting for at least 2-6 week ${ }^{1-3}$. Histopathologically, COM is defined as irreversible mucosal changes within the middle ear cavity ${ }^{4}$. It is estimated that COM affects 65 million to 330 million people worldwide, and around $60 \%$ of them show clinically significant hearing impairment ${ }^{5}$. In COM patients, hearing impairment is often a permanent complication, due to irreversible tissue alternations in the middle ear cleft. It has been suggested that the degree to which hearing is compromised is proportional to the damage caused to the structures of the middle ear. The hearing thresholds may be influenced by various factors including the size and location of the tympanic membrane perforation, the ossicular chain erosion or discontinuity as well as the presence of cholesteatoma and its growth patterns ${ }^{6-8}$. One of the most consequences of COM is its impact on three tiny bones known as the ossicles or the ossicular chain ${ }^{9,10}$. It has been shown that ear ossicles transmit the sound-induced vibrations of the tympanic membrane to the cochlea via the oval window (sound transmission role). Instead of being attached to other bones, the ossicular chain is suspended within the middle ear cavity by ligaments and tendons, as well as its attachments to the tympanic membrane and the oval window ${ }^{11}$. Another function of the ossicles is to apply force to one window only of the cochlea (sound amplification role). If the ossicles were missing, and the pressure of the incoming sound wave was applied equally to both windows, there would be a reduced flow of cochlear fluids $^{12}$. The Pure-Tone Average (PTA) and Air-Bone Gap (ABG) preoperative findings in patients affected by COM have been suggested as a useful method to predict ossicular chain condition. In a study conducted by Carrillo et al..$^{10}$ the predictive value of $A B G$ levels for presence of a significant ossicular chain disruption in the patients with COM was evaluated. It was found that low $A B G$ at low frequencies is the suggestive of absence of ossicular chain and extensive $A B G$ at high frequencies proposes the presence of ossicular chain. The aim of the present study to determine the prevalence and type of ossicular defects in patients with COM and to correlate the ossicular chain status with clinical parameters, and hearing function ${ }^{11}$.

\section{MATERIALS AND METHODS}

Participants: This retrospective clinical study was conducted on COM patients submitted to surgery in the Otology Clinic at Imam Khomeini Hospital, Ahvaz Jundishapur University of Medical Sciences (AJUMS), Iran. The study population composed of 107 patients (52 males; 55 females), age ranged from 18 to 75 years (mean age: 33.34 ). The patients were diagnosed as COM with anamnesis and micro-otoscopy examination. The individuals with a history of previous ear surgery, ear trauma, congenital cholesteatoma and previous radiation treatment were excluded. Patients with systemic diseases such as diabetes mellitus and renal failure were also excluded. All experimental procedures were approved by the Ethics Committee of AJUMS, Ahvaz, Iran (Registration code: 3293). Written informed consent was obtained from all participants to take part in this study.

Procedures: All participants were evaluated with computed tomography of the temporal bone and pure tone audiometry. Audiometry was carried out in a soundproof chamber using AC 40 dual channel Audiometer (Intracoustics Co.Denmark). The hearing thresholds were assessed over the frequency ranges of 250 to $8000 \mathrm{~Hz}$ for Air Conduction (AC) and 500 to 4000 $\mathrm{Hz}$ for Bone Conduction (BC) pathways. The Pure-Tone Average (PTA) and Air-Bone Gap (ABG) average were calculated using frequencies 500,1000 and $2000 \mathrm{~Hz}$. Intraoperative microscopic evaluation of ossicular status chain was carried out by an experienced surgeon in order to determine the presence and site of lesion of each ossicle. For each patient, the integrity of the ossicular chain and tympanic membrane status was also recorded.

Statistical analysis: The descriptive analysis of data was performed through the calculation of frequency and percentage and the drawing of frequency distribution table. The independent sample t-test was used to test the differences of means between two groups. The Chi-square test was used to compare the groups for categorical variables. Data were analyzed with statistical package SPSS (version 16). All statistical tests were two-sided, and $p<0.05$ was considered as statistically significant.

\section{RESULTS}

COM in 33 patients (27.3\%) was observed in the right ear, in 45 patients $(41.28 \%)$ in the left ear and in 31 patients $(28.45 \%)$ in both ears. Among the overall patients affected by COM, tympanic membrane perforation was peripheral in 28 patients (25.69\%), central in 56 patients $(51.37 \%)$ and total in 25 patients $(22.94 \%)$. The primary complaint of the patients was "hearing loss", observed in $85.04 \%$ (91/107) of the cases. Table 1, reveals the distribution frequencies of damage to the middle ear ossicles according to the site of injury. Our findings demonstrated that the malleus was the most resistant ossicle to erosion in COM. It was found intact in $70(65.42 \%)$ and abnormal in $29.58 \%$ of the participants. The "manubrium" was the most commonly necrosed part of the malleus and was found eroded in $16(14.95 \%)$ of the participants. Incus was the most commonly abnormal ossicle found in our study (Table 1). We found that the incus was intact only in $33(30.84 \%)$ patients, and abnormal in $74(69.16 \%)$ cases. The most commonly necrosed parts of the incus were the "long + short process" in $29(19.33 \%)$ and the long process in $25(16.67 \%)$ of the patients. We also found that stapes was intact in $54(50.46 \%)$ cases while in $53(49.54 \%)$, the 
Table 1: Distribution of lesions in middle Ear ossicles.

\begin{tabular}{|c|c|c|c|c|c|}
\hline \multicolumn{6}{|c|}{ Middle Ear Ossicle } \\
\hline Malleus & n (\%) & Incus & n (\%) & Stepes & n (\%) \\
\hline Intact & $70(5.42)$ & Intact & $33(30.84)$ & Intact & $54(50.46)$ \\
\hline Manubrium & $16(4.95)$ & Body & $10(9.34)$ & Footplate & $14(13.08)$ \\
\hline Head & $6(5.51)$ & Long process & 21(19.62) & Cura & $14(13.08)$ \\
\hline Manubrium + Head & $5(4.67)$ & Short + Long process & $24(22.42)$ & Footplate + Cura & 25 (23.36) \\
\hline Absent & $10(9.34)$ & Absent & $19(17.75)$ & & \\
\hline
\end{tabular}

Table 2: The mean pure tone average and air bone gap values in intact and damaged ossicular chain patients

\begin{tabular}{cccc}
\hline Index & \multicolumn{2}{c}{ Ossicular chain status } & p-value \\
\cline { 2 - 3 } & Intact & Damaged & $>0.05$ \\
Pure Tone Average (PTA) & $44.75 \pm 9.40$ & $48.39 \pm 13.17$ & $>0.05$ \\
Air Bone Gap (ABG) & $32.76 \pm 14.34$ & $36.25 \pm 10.51$ & \\
\hline
\end{tabular}

stapes was found to be affected by the disease (Table 1). The malleoincudal joint was observed intact in 72 $(67.29 \%)$ and discontinuous in $36(32.71 \%)$ patients. The incudostapedial joint was intact in $63(58.88 \%)$ patients and discontinuous in $44(41.12 \%)$ cases of disorder. Furthermore, the ossicular chain was found intact in 59 (55.14\%) subjects. Although discontinuous ossicular chain group displayed higher PTA and ABG values than intact group, this difference was not significant (Independent sample t-test, $p>0.05$ ). Chi-square test did not show significant differences between ossicular chain status (damaged/intact) and sex variables $(p>0.05)$. Table 2 shows the mean PTA and ABG values in "intact" and "discontinuous" ossicular chain groups.

\section{DISCUSSION}

The purpose of our study was to evaluate middle ear exploration findings, and frequency of ossicular chain anomalies in COM patients. Conductive hearing loss $(\mathrm{CHL})$ following COM may be attributed to factors such as tympanic membrane perforation, ossicular chain disruption, or middle ear space inflammation. It is well documented that long-term sensory deprivation resulting from $\mathrm{CHL}$ will produce irreversible changes in the anatomical and functional integrity of the central auditory system structures ${ }^{13-17}$ which may lead to the outbreak of language, cognitive, psychological and emotional problems in person ${ }^{18,19}$. In COM cases, infection through bone erosion can also be spared out in mastoid cortex, petrous part of the temporal bone, facial nerve, lateral sinus and dura matter. Since the middle ear is on the vicinity of the brain and only a thin bone separates it from the brain cavity, the investigation and evaluation of clinical and pathological condition of the patient has a particular importance7 our results exhibited that the malleus was the most resistant ossicle in cases of COM, found normal in 70 (65.42\%) patients. It was eroded in $27(25.24 \%)$ and absent in $10(9.34 \%)$ cases. However, Varshney et al. ${ }^{20}$ found a higher incidence of intact malleus (80.67\%) during their study on adult's population with COM. The "manubrium" was the most commonly necrosed part of the malleus and was found eroded in 16 (14.95\%) of our participants. Varshney et al. ${ }^{20}$ findings also showed that the manubrium was the most commonly necrosed component of ossicle, damaged in $19(12.66 \%)$ patients. We observed that incus was intact in 33 (30.84\%) patients, necrosed in 55 $(51.41 \%)$ cases and absent in ${ }^{19}(17.75)$ cases. This finding demonstrates that incus could be regard as the most common ossicle to get necrosed in patients with COM. In a similar investigation, Udaipurwala, et al. ${ }^{21}$ and Varshney et al. ${ }^{20}$ reported lower incidence of necrosis of the incus at $41.00 \%$ and $21.34 \%$, respectively. The long process of incus was found to be the most commonly necrosed component of ossicle affected in 45 (42.05\%) patients. In our sample, 53 of 107 enrolled patients $(49.54 \%)$ revealed some type of stapedial necrosis, which is higher than the incidences of reported by Udaipurwala et al. ${ }^{21}$ and Sade $(36 \%)^{22}$. Intraoperative inspection exhibited intact malleoincudal and incudostapedial joints in 72 (67.29\%) and $63(58.88 \%)$ of patients, respectively. These findings are in agreement with those of Varshney et al. ${ }^{20}$ study. Carrillo et al. ${ }^{10}$ reported that the ABG in patients affected by $\mathrm{COM}$ has been related to ossicular chain status so that smaller value of $A B G$ would predict ossicular integrity, whereas larger values of $A B G$ (especially at high frequencies) would suggest ossicular discontinuity. In our investigation, we did not found significant $A B G$ and PTA differences between "intact ossicular" and "disrupted ossicular" groups. Hence, we believe that the ABG and PTA values do not always show the real state of the middle ear transmission system. In our study, there was no statistical correlation between patients ' gender and ossicular condition (intact/disrupted). This finding is in accordance with Nemati et al. results ${ }^{23}$ who reported no significant relationship between gender and ossicular chain dislocation, and stapes footplate thickness

\section{CONCLUSION}

Our results demonstrated that ossicular chain abnormality is a prevalent condition in patients with COM. Incus was the most susceptible ossicle to erosion in COM, whereas the malleus was the most resistant. Furthermore, ABG 
and PTA values cannot be considered as a potential preoperative predictor for ossicular chain status.

\section{Acknowledgements}

This paper was result of an M.D. thesis in Medical School at Ahvaz Jundishapur University of Medical Sciences (AJUMS), Ahvaz, Iran. The project was funded by the Research Deputy of AJUMS (registration number: 3293).

\section{Conflict of Interest}

No potential conflict of interest relevant to this article was reported.

\section{REFERENCES}

1. Roland PS. Chronic suppurative otitis media: A clinical overview. Ear Nose Throat J. 2002;81 (8 Suppl 1):8-10.

2. Verhoeff M, Vander Veen EL, Rovers MM, Sanders EA, Schilder AG. Chronic suppurative otitis media: a review. Int J Pediatr Otorhinolaryngol. 2006;70:1-12.

3. Sun J, Sun J. Intracranial complications of chronic otitis media. Eur Arch Otorhinolaryngol. 2014;271(11):2923-6.

4. Merchant SN. Scott Brown's Otolaryngology, Head Neck Surgery: 7th edition. 2011;93(7):559.

5. Maruthy S, Mannarukrishnaiah J. Effect of early onset otitis media on brainstem and cortical auditory processing. Behav Brain Funct. 2008;4:1-17.

6. Mostafa BE, EliFiky LM, Eli-Sharnouby MM. Complications of suppurative otitis media: still a problem in the 21st century. $J$ Otorhinolaryngol Relat Spec. 2009;71(2):87-92.

7. Dubey SP, Larawin V. Complications of chronic suppurative otitis media and their management. Laryng. 2007;117(2):264-67.

8. Silveira Netto LS, DaCosta SS, Sleifer P, Braga ME. The impact of chronic suppurative otitis media on children's and teenagers hearing. Int J Pediatr Otorhinolaryngol. 2009;73:175-6.

9. Ebenezer J, Rupa V. Preoperative predictors of incudal necrosis in chronic suppurative otitis media. Otolaryngol Head Neck Surg. 2010;142(3):415-20.

10. Carrillo RJC, Yang NW, Abes GT. Probabilities of ossicular discontinuity in chronic suppurative otitis media using pure tone audiometry. Otol Neurotol. 2007;28(8):1034-37.
11. Gelfand SA. Essentials of audiology. 4th edition. Thieme Publisher. 2012: 1-37.

12. Pickles JO. In introduction to the physiology of hearing. 4th edition. J Emerlad Publisher. 2012: 15-16.

13. Albera R, Canale A, Piumetto E, Lacilla M, Dagna F. Ossicular chain lesions in cholesteatoma. Acta Otorhinolaryngol. 2012;32(5):30913.

14. Saki N, Samarbaf Zadeh AR, Sheikhpour JR, Noori SM, Kayedani GA. Prevalence rate of Helicobacter pylori infection in chronic otitis media with effusion patients. Jundishapur $\mathrm{J}$ Microbiol. 2014;7(3):156-94.

15. Popscu MV, Polley DB. Monaural deprivation disrupts development of binaural selectivity in auditory midbrain and cortex. J Neuron. 2010;65:718-31.

16. Tucci DL, Cant NB, Durham D. Effects of conductive hearing loss on gerbil central auditory system activity in silence. Hear Res. 2001;155(2):124-32.

17. Bayat A, Farhadi M, Emamdjomeh H, Saki N, Mirmomeni G, Rahim F. Effect of conductive hearing loss on central auditory function Braz J Otorhinolaryngol. 2017;83(2):137-41.

18. Winskel $\mathrm{H}$. The effects of an early history of otitis media on children's language and literacy skill development. $\mathrm{Br} \mathrm{J}$ Educ Psychol. 2006;76(4):727-44.

19. Nittrouer S, Burton LT. The role of early language experience in the development of speech perception and phonological processing abilities: evidence from 5-year-olds with histories of otitis media with effusion and low socioeconomic status. J Com Disord. 2005;38(1):29-63.

20. Varshney S, Nangia A, Bist SS, Singh RK, Gupta N. Ossicular chain status in chronic suppurative otitis media in adults. Indian $J$ Otolaryngol Head Neck Surg. 2010;62(4):421-6.

21. Udaipurwala IH, Iqbal K, Saqulain G, Jalisi M. Pathlogical profile in chronic suppurative otitis media in the regional experience. J Pak Med Assoc. 1994;44(10):235-7.

22. Sade J, Berco E, Buyanover D, Brown M. Ossicular damage in chronic middle ear inflammation. Acta Otolaryngol. 1981;92:273-83.

23. Nemati S, Ebrahim N, Kaemnejad E, Aghjanpour M, Abdollahi O. Middle ear exploration results in suspected otosclerosis cases: are ossicular and footplate area anomalies rare? Iran J Otorhinolaryngol. 2013;25(72):155-60. 